\title{
The Role of the Pie-Crusting Technique of the Medial Collateral Ligament in the Arthroscopic Inside-out Technique for Medial Meniscal Repair With or Without Anterior Cruciate Ligament Reconstruction: A Satisfactory Repair Technique
}

\author{
Mehmet Erdem, M.D., \\ Levent Bayam, M.R.C.S.(Ed), M.Ch.(T\&O, UK), M.Phil.(UK), M.Sc., F.E.B.O.T., \\ Ahmet Can Erdem, M.D., Deniz Gulabi, M.D., Abdulhalim Akar, M.D., and \\ Alauddin Kochai, M.D.
}

\begin{abstract}
Purpose: To assess the results of a technique for pie crusting of the medial collateral ligament (MCL) and inside-out medial meniscal repair and perform a comparison with the literature. Methods: This retrospective study consisted of electronic data collection between 2012 and 2017 with a minimum of 2 years' follow-up. The inclusion criteria were the presence of difficult medial meniscal tears with joint tightness requiring pie crusting and the presence of acute or chronic tears of zone I or II with or without anterior cruciate ligament reconstruction (ACLR) using hamstring autograft during the same session. The primary outcome of the study was the achievement of good results with the aforementioned technique. There was no control group. All patients underwent assessments at 1, 2, and 6 months in outpatient clinics. At 6 months, the Lysholm knee score was calculated. For statistical analysis, the Social Science Statistics online program was used to perform descriptive analysis and assess any associations between the variables. Results: This study included 53 patients from a single surgeon's practice; of these patients, 31 underwent additional ACLR using hamstring autograft during the same session. The mean age was 29.43 years (range, 14-49 years), and the mean increase in the medial joint space width was $3.21 \mathrm{~mm}$ (range, $2-5 \mathrm{~mm}$ ) with pie crusting. At 6 months, the average Lysholm score was 93 (range, 67-100) and the average visual analog scale score was 0.8 (range, $0-4$ ). There was no meaningful association between age, tear pattern, chronicity of tear, joint space width obtained after pie crusting, and associated anterior cruciate ligament tear. Patients returned to their daily activity level at 4.5 months on average and returned to sporting activities at 7.4 months on average. Saphenous nerve symptoms were observed in 5 patients, but no infection or instability was documented in the follow-up period. Conclusions: In this study, we obtained good outcomes using arthroscopic inside-out medial meniscal repair combined with pie crusting for the release of the MCL, with or without ACLR. Level of Evidence: Level IV, therapeutic case series.
\end{abstract}

$\boldsymbol{S}^{\mathrm{n}}$ tudies in the literature have suggested that meniscal repair has a positive effect on osteoarthritis development in the long term compared with partial meniscectomy because repair may prevent

From Medical School, Sakarya University, Sakarya, Turkey (M.E., L.B., A.A., A.K.); Bezmialem Vakif University, Istanbul, Turkey (A.C.E.); and Orthopaedic Department, Bahcesehir University, Istanbul, Turkey (D.G.).

The authors report no conflicts of interest in the authorship and publication of this article. Full ICMJE author disclosure forms are available for this article online, as supplementary material.

The abstract was presented at the following meetings: XIV.Türk Spor Yaralanmalari Artroskopi Ve Diz Cerrahisi (TUSYAD) Kongresi, Istanbul, Turkey, 2018; 12th International Congress of ISKSAA (International Society for Knowledge for Surgeons on Arthroscopy and Arthroplasty), Leeds, cartilage degeneration that occurs after meniscectomy. ${ }^{1,2}$ The described techniques have included inside-out, outside-in, and all-inside techniques. Insideout and all-inside repairs are most commonly used

\footnotetext{
England, 2018; and SICOT (Société Internationale de Chirurgie Orthopédique et de Traumatologie), Montreal, Canada, 2018.

Received February 4, 2020; accepted August 18, 2020.

Address correspondence to Levent Bayam, M.R.C.S.(Ed), M.Ch.(TeO, UK), M.Phil.(UK), M.Sc., F.E.B.O.T., Medical School, Sakarya University, Adnan Menderes Caddesi 54100, Sakarya,Turkey.E-mail:levbayam@hotmail.co.uk

(C) 2020 THE AUTHORS. Published by Elsevier Inc. on behalf of the Arthroscopy Association of North America. This is an open access article under the CC BY-NC-ND license (http://creativecommons.org/licenses/by-nc-nd/4.0/). 2666-061X/2069

https://doi.org/10.1016/j.asmr.2020.08.005
} 
today. ${ }^{1,3}$ To overcome iatrogenic damage to the articular cartilage due to a narrow joint space, especially in arthroscopic repair of the posterior horn, one of the described surgical techniques is pie crusting, which involves repetitive puncturing of the MCL with a needle in valgus stress, thereby facilitates expansion of the medial joint space, allows access to the medial meniscus, provides better visualization and an adequate working space. ${ }^{3,4}$ Pie crusting of the MCL is a transcutaneous release that facilitates expansion of the medial joint space and allows access to the medial meniscus to provide better visualization and an adequate working space. ${ }^{3,4}$ Therefore, this could be an advantage to ease the surgical approach, with better handling of the repair and a decrease in chondral damage. ${ }^{4}$ Previous studies have shown that pie crusting is an effective and safe procedure and the MCL heals fully with no residual valgus laxity, in contrast to the lack of healing capability of the articular cartilage once injured during arthroscopic procedures. ${ }^{4-6}$

Pie crusting is a well-known technique used in both total knee replacement and meniscal repair but is probably less commonly performed in the latter technique. The purpose of our study was to assess the results of a technique for pie crusting of the MCL and inside-out medial meniscal repair and perform a comparison with the literature. Our hypothesis was that we would obtain good outcomes in patients by use of the described technique by improving visualization.

\section{Methods}

This retrospective study, with ethical approval from the local committee, consisted of data collection between 2012 and 2017 with a minimum of 2 years' follow-up. The data were collected from the electronic medical records, and the measurements of medial joint space width (JSW) were calculated carefully from the intraoperatively recorded arthroscopic video footage for each patient.

The inclusion criteria were the presence of medial meniscal tears and the presence of acute or chronic tears of zone I or II (red-red or red-white zone) with or without anterior cruciate ligament reconstruction (ACLR) using hamstring autograft during the same session. The indications for pie crusting by the senior surgeon (M.E.) were the presence of a tight medial joint space and difficult cases such as posterior horn tears or tears with extension to the midsubstance, large longitudinal midsubstance tears, and buckle-handle tears, which require expansion of the JSW to avoid iatrogenic chondral damage. The exclusion criteria were medial meniscal repairs without pie crusting and patients with insufficient data or lack of intraoperative arthroscopic videos. The primary outcome of the study was the achievement of good results with the aforementioned technique. There was no control group.

\section{Surgical Technique}

Initial diagnostic arthroscopy was performed using anterolateral and anteromedial portals after the patient was positioned supine with the leg dangling down. Granulation tissue debridement on both sides of the tear was performed with a meniscal rasp (Acufex; Smith \& Nephew, Andover, MA) or, alternatively, a 3.5-mm full-radius shaver.

Pie crusting was applied to selected patients with a narrow JSW (Fig 1), which was not wide enough to perform meniscal repair without iatrogenic damage in the senior surgeon's experience. Pie crusting was performed at $15^{\circ}$ to $20^{\circ}$ of knee flexion and with the application of gentle valgus stress. It involved repetitive puncturing (3-5 times) of the MCL percutaneously from anterior to posterior using an 18-gauge needle superior to the medial joint line (Fig 2), by observing the needle tip through arthroscopy (Fig 3), from just above the level of the meniscocapsular junction, proximal to the joint line, until the desired gap was obtained (Fig 4).

When a patient underwent ACLR during the same session, anterior cruciate ligament (ACL) tunnels were initially prepared; the meniscus was then repaired before ACL graft (hamstring tendon) insertion. Meniscal suturing was performed without tying (Fig 5). Once the ACLR was completed, these meniscal stitches were then tied over the capsule, medially or posteromedially (Fig 6). Zone-specific cannulas were used with long and flexible needles to pass sutures through the joint into the meniscal substance under an arthroscopic view. The stitches were tied carefully without hamstring entrapment, and a small posterior joint line incision with a length of approximately $2 \mathrm{~cm}$, posterior to the MCL, was used to retrieve the sutures and tie them directly on the capsule (Fig 6); another skin incision was added

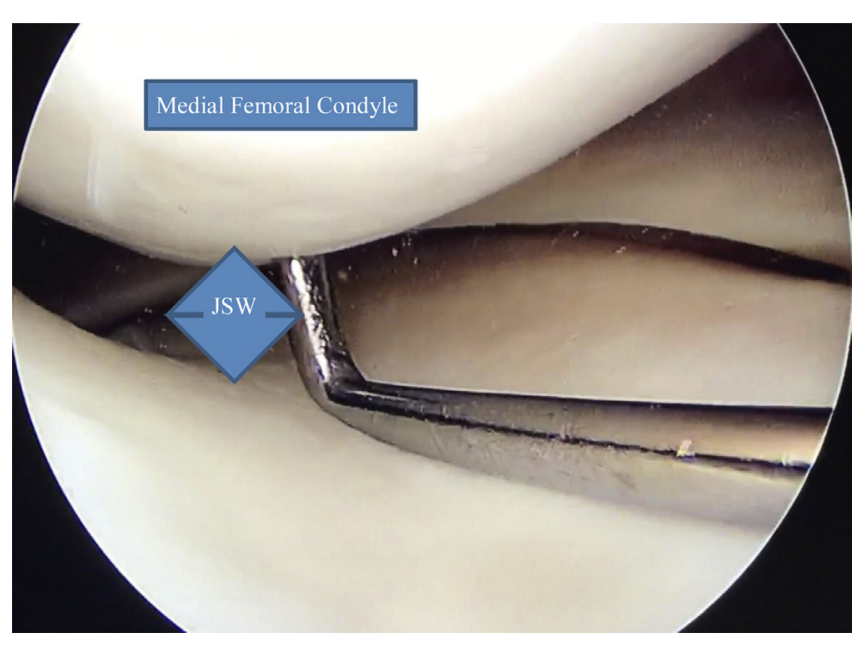

Fig 1. Arthroscopic view of the knee via the lateral portal showing the tip of the hook and a narrow medial joint space to perform meniscal repair (left side). (JWS, joint space width.) 


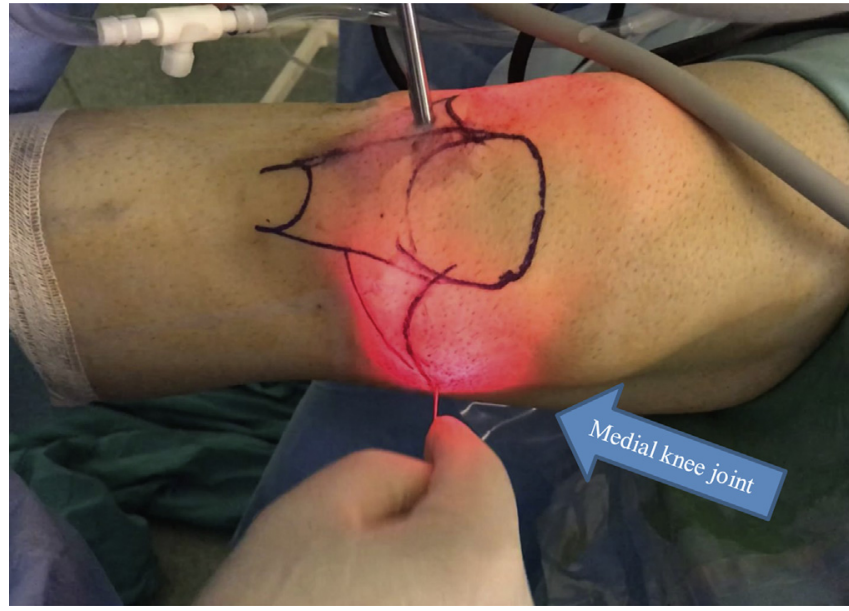

Fig 2. Repetitive puncturing of the knee medial collateral ligament (pie crusting) during arthroscopy to expand the medial joint space width. The patient is in the supine position with the leg dangling down.

if necessary. For patients without ACLR, a microfracture area was formed in the lateral wall of the femoral notch to promote healing via bone marrow medullary progenitor cell release.

\section{Postoperative Protocol}

Initial postoperative rehabilitation for meniscal repair alone and for meniscal repair combined with ACLR was the same and followed the local physiotherapist's protocol. On the first day postoperatively, patients started isometric quadriceps exercises, and during the first 4 weeks, flexion of the knee up to $90^{\circ}$ was allowed with an adjustable brace, followed by full flexion gradually. Closed-chain exercises were started after 4 weeks and continued with the addition of open-chain exercises after 6 weeks. In the first 6 weeks, no weight bearing was allowed except toe-touch weight bearing; later, gradual weight bearing was allowed, with full weight bearing permitted at 10 weeks.

All patients underwent assessments at 1, 2, and 6 months in outpatient clinics. At 6 months, the Lysholm knee score and visual analog scale (VAS) pain score were calculated. After the first 6 months, patients underwent regular follow-up every 6 months. During all follow-up visits, range of movement (ROM) of the knees was measured and complications, if any, were documented.

\section{Medial JSW Measurement}

Intraoperative arthroscopic video footage was used to calculate the medial JSW and to assess the amount of increase in the JSW after pie crusting in the postoperative period as part of the study. The available probes in the theater setting were all Acufex probes (Smith \& Nephew), and the tips of all probes measured $3 \mathrm{~mm}$. The measurement was performed at the

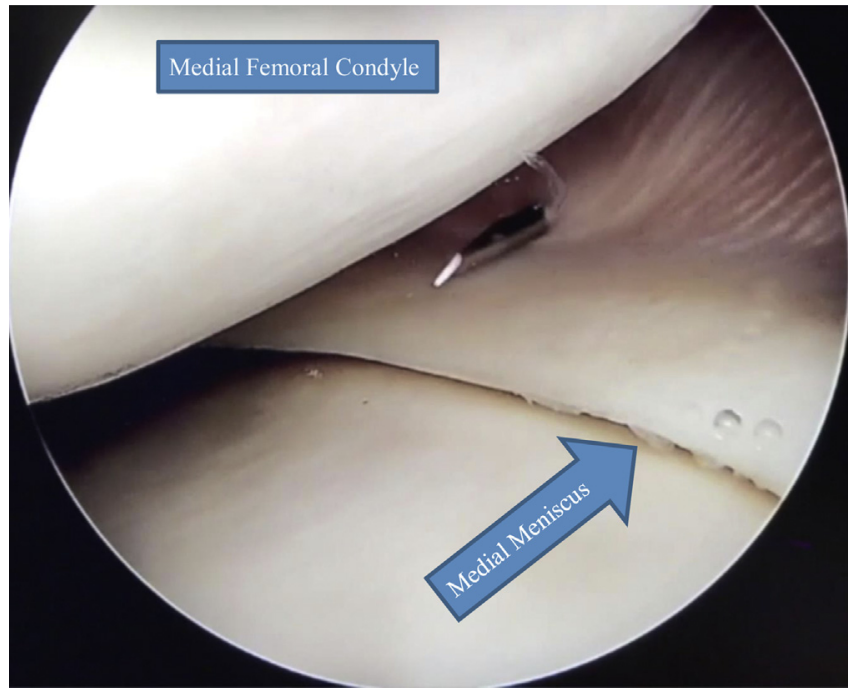

Fig 3. Intraoperative arthroscopic medial knee view via the lateral portal showing the tip of an 18-gauge needle for pie crusting.

narrowest point between the 2 condyles (femur and tibia) in the medial compartment of the knee from the static images.

\section{Data Analysis}

For statistical analysis, the Social Science Statistics online program was used to perform descriptive analysis and to assess any associations between variables. The associations between age, tear pattern, chronicity of tear, JSW obtained after pie crusting, and associated ACL tear were analyzed. To assess the associations between number of stitches, tear length, Lysholm and VAS scores, ROM, and increase in joint space, correlation tests were used. The Pearson correlation test was used for parametric data; in addition, to assess the

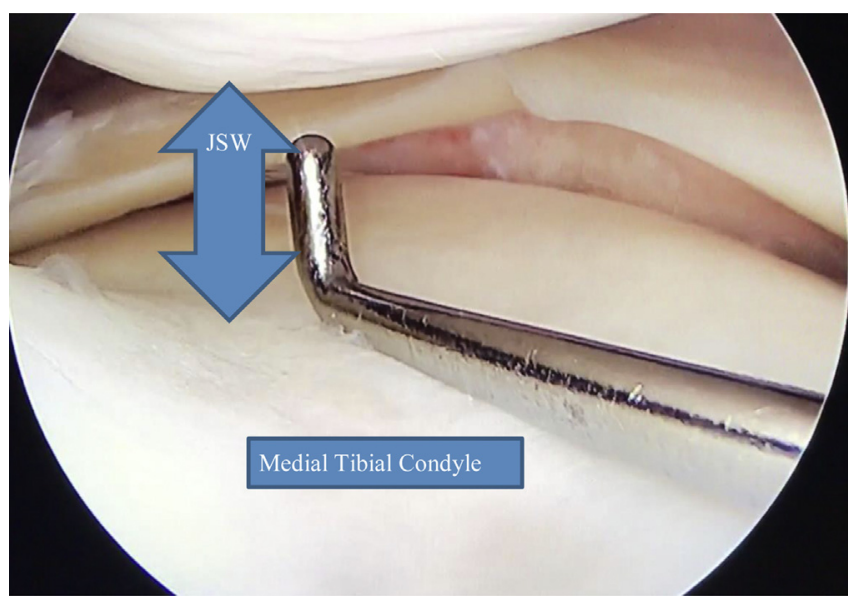

Fig 4. Arthroscopic view of the medial knee compartment via the lateral portal with increased joint space width (JWS) after pie crusting. 


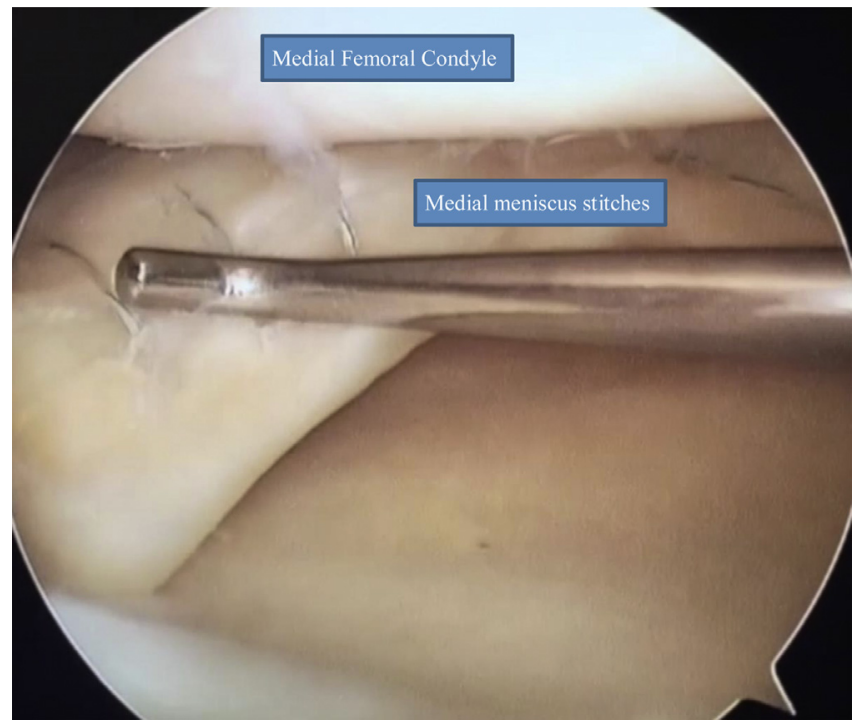

Fig 5. Arthroscopic view of the medial knee compartment showing mainly the posterior horn to midsubstance of the medial meniscus via the lateral portal after meniscal repair.

associations between the other variables, the Spearman $\rho$ value was used.

\section{Results}

The study included 53 patients (49 male and 4 female patients) from a single surgeon's practice; of these patients, 31 underwent additional ACLR using hamstring autograft during the same session. The mean age was 29.43 years (range, 14-49 years), and the mean followup period was 31.6 months (range, 24-45 months; minimum, 2 years). Acute meniscal injuries (within 3 months) were observed in 18 patients, whereas the remainder of patients $(n=35)$ had chronic injuries. We noted buckle-handle tears in 17 patients and radial tears in 7 , with longitudinal tears in the remainder $(\mathrm{n}=$ $29)$. Medial meniscal tears mostly involved the posterior horn, and 35 of 53 patients had posterior horn tears. Sixteen patients had posterior horn tears involving the midsubstance, 19 had posterior horn tears only, and 18 had midsubstance tears. The meniscal tear was in the red-white zone in 22 patients and in the redred zone in the remainder. The mechanism of injury is shown in Table 1 . Most of the patients were injured during football, with the study comprising predominantly male patients. Different mechanisms were noted among the 4 female patients (fall in 2, judo in 1, and squatting in 1) (Table 1).

With the pie-crusting technique, there was a mean increase in the JSW of $3.21 \mathrm{~mm}$ (range, 2-6 mm) (Fig 4, Table 2). The mean number of stitches was 5.7 (range, 2-13) (Fig 3). At 6 months, the average Lysholm score was 93 (range, 67-100) and the average VAS score was 0.8 (range, $0-4$ ). Most patients returned to their daily activity level (which constituted a return to normal lifestyle activities such as walking, as well as a return to work) at 4 months (range, 3-6 months; mean, 4.5 months; median, 4 months; mode, 4 months; standard deviation, 0.96 months), whereas the return to sporting activities took longer than 6 months, occurring at a mean of 7.4 months (range, 6-12 months; standard deviation, 1.85 months).

There was no documentation of infection, but saphenous nerve symptoms were observed in 5 patients; all these symptoms were resolved during followup. No instability was detected in the clinical assessment. In the assessment of the intraoperative video footage, we did not identify any iatrogenic cartilage injury.

No correlation was found between tear zone and return to sporting activities, between saphenous nerve symptoms and number of stitches, or between Lysholm score and ACLR having been performed (Table 3). However, there was a strong correlation between the number of stitches and the tear length (Table 4, Fig 4). We found a moderate correlation between the Lysholm and VAS scores, a moderate correlation between the Lysholm score and ROM, and a weak correlation between the number of stitches and the increase in JSW via pie crusting (Table 4). One patient had a recurrent meniscal tear after 2 years and eventually underwent partial meniscectomy. No meaningful association was observed between age, tear pattern, tear chronicity, JSW obtained after pie crusting, and associated ACL tear using Spearman $\rho$ values (tear pattern and tear chronicity, $\rho=0.75$; tear pattern and postoperative JSW, $\rho=0.7$; postoperative JSW and ACL tear [or no ACL tear], $\rho=0.59$; age and tear pattern, $\rho=0.15$; age and tear chronicity, $\rho=0.33$; and age and JSW, $\rho=$ $-0.08)$.

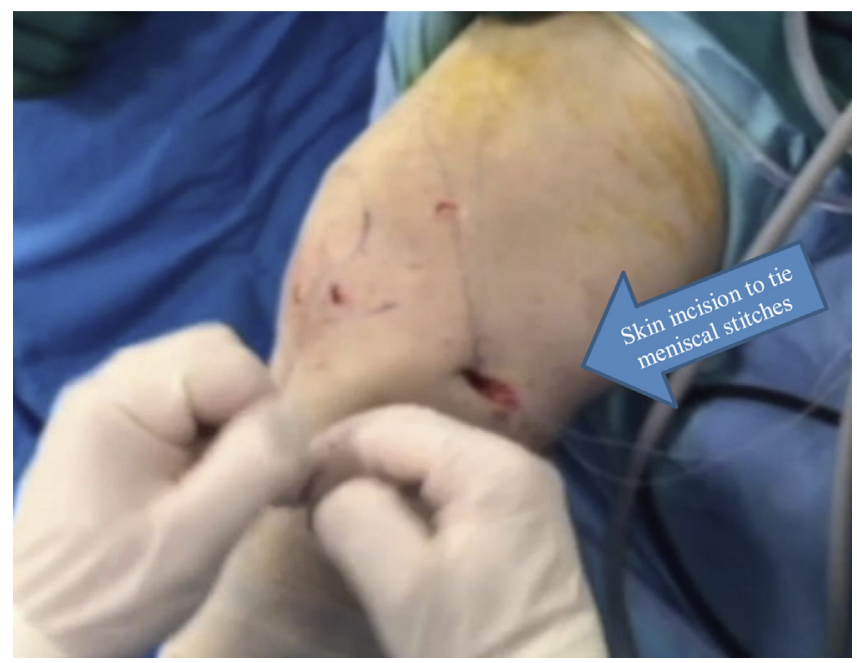

Fig 6. Posteromedial skin incision to tie medial stitches over the capsule. The patient is in the supine position with the leg dangling down. 
Table 1. Mechanism of Injury

\begin{tabular}{lccccc}
\hline & \multicolumn{5}{c}{ Mechanism of Injury } \\
\cline { 2 - 6 } & Football & Fall & Squatting & Basketball & Judo \\
\hline Patients, n & 44 & 6 & 1 & 1 & 1 \\
\hline
\end{tabular}

\section{Discussion}

In this study, we observed good outcomes and low complication and failure rates after medial meniscal repair with an inside-out repair and pie crusting of the MCL in the absence of a control group. In the assessment of the intraoperative video footage, we did not detect any iatrogenic cartilage injury. No meaningful association was found between age, tear pattern, chronicity of tear, JSW obtained after pie crusting, and associated ACL tear.

We used a 3-mm probe to estimate the medial JSW initially and after pie crusting using intraoperative arthroscopic video footage. Previously, arthroscopic video clips were used to estimate the extent of chondropathy, ${ }^{7}$ and the intrarater and inter-rater reliability of estimating cartilage lesion size with arthroscopic views was found to be moderate to good using a 3-mm probe. ${ }^{8}$ The main indication or reason for pie crusting is adequate visualization, especially in patients with tight medial compartments, ${ }^{9}$ thereby avoiding an iatrogenic chondral injury because of the narrow joint space. How much the compartment space can be expanded or needs to be expanded is questionable. Increasing the space too much may lead to instability, in theory, and not increasing the space enough may be a hindrance to the surgical technique. Therefore, there should be a balance in between increasing the joint space too much and too little. We were not able to find any literature on the minimum JSW required to perform meniscal repair, but the normal value of minimum JSW is a mean of $4.8 \mathrm{~mm}$ for healthy women and $5.7 \mathrm{~mm}$ for healthy men. ${ }^{10}$ The main indication for pie crusting was to achieve adequate visualization-especially with tight medial compartments ${ }^{4}$-and, thereby, to avoid an iatrogenic chondral injury. In the literature, the expansion has been described mostly as measuring between 3 and $5 \mathrm{~mm}$. $^{9,11}$ Similarly, in our study, the mean increase in the JSW with pie crusting was $3.21 \mathrm{~mm}$ (range, $2.5 \mathrm{~mm}$ ), and

Table 2. Number of Stitches in Medial Meniscal Repair and Medial Joint Space Width Preoperatively and Postoperatively (After Pie-Crusting Procedure)

\begin{tabular}{lccc}
\hline & $\begin{array}{c}\text { No. of } \\
\text { Stitches }\end{array}$ & $\begin{array}{c}\text { Preoperative } \\
\text { Joint Space } \\
\text { Width, mm }\end{array}$ & $\begin{array}{c}\text { Postoperative Joint } \\
\text { Space Width, mm }\end{array}$ \\
\hline Mean & 5.7 & 4.61 & 7.82 \\
Median & 6 & 5 & 8 \\
Mode & 6 & 5 & 7 \\
SD & 2.44 & 0.5 & 0.85 \\
\hline
\end{tabular}

Table 3. Spearman Correlation for Nonparametric Values

\begin{tabular}{|c|c|c|c|}
\hline $\begin{array}{l}\text { Spearman } \\
\text { Correlation }\end{array}$ & $\begin{array}{l}\text { Return to Sport } \\
\text { and Tear Zone } \\
\text { (R-R or R-W) }\end{array}$ & $\begin{array}{l}\text { Saphenous } \\
\quad \text { Nerve } \\
\text { Symptoms and } \\
\text { No. of Stitches }\end{array}$ & $\begin{array}{l}\text { Lysholm Score } \\
\text { and ACLR } \\
\text { Performed }\end{array}$ \\
\hline$R$ value & -0.007 & 0.366 & 0.135 \\
\hline$P$ value & .975 & $>.05$ & .486 \\
\hline
\end{tabular}

after the pie-crusting procedure, the mean JSW measured $7.82 \mathrm{~mm}$. Previous studies have shown that pie crusting is an effective and safe procedure and that the MCL heals fully with no residual valgus laxity. ${ }^{4-6}$ The selective medial pie-crusting technique has also been declared an effective and safe method to obtain a balanced mediolateral gap in primary total knee arthroplasty. ${ }^{11}$

Current arthroscopic techniques include inside-out, outside-in, and all-inside repairs, and each all-inside technique has its indications, advantages, and disadvantages. ${ }^{12}$ No differences in meniscal healing, clinical failure rate, or outcome have been shown between inside-out and all-inside meniscal repair techniques. ${ }^{3,12}$ According to Nelson and Bonner, ${ }^{12}$ the inside-out technique is quite versatile; moreover, it allows for fine precision and can be used for most repairable tear patterns involving the posterior horn or body of the meniscus. ${ }^{13}$ The inside-out meniscal repair technique has been declared the standard of care for meniscal repair and has shown improved patient outcomes; this technique is best used for posterior horn, middle-third, peripheral capsule, and bucket-handle tears. ${ }^{13,14}$ The drawbacks of this technique include the need for additional incisions, the risk of neurovascular injury, the need for an assistant, and theoretically, an increased procedure time. More nerve symptoms were reported in association with the inside-out repair, and more implant-related complications were associated with the all-inside technique. ${ }^{13}$ Grant et al. ${ }^{3}$ reported that the prevalence of nerve injury or irritation was $9 \%$ with the inside-out technique. In our study, saphenous nerve symptoms developed in 5 patients $(9.43 \%)$; all these symptoms were resolved during the follow-up period. The mean number of stitches for meniscal repair was variable in the literature, between 2 and $12 .{ }^{14-16}$ On average, 10 to 12 sutures were used in a study by Maffulli et al., ${ }^{14}$ and 5 were used by EspejoReina et al. ${ }^{15}$ We were not able to find studies reporting the effect of the number of stitches on healing. In our study, an average of 5.7 stitches per tear (range, 213 stitches per tear) were used.

Failure of meniscal repair after 2 years was not infrequent. Some studies have reported failure rates of 
Table 4. Pearson Correlation for Parametric Values

\begin{tabular}{|c|c|c|c|c|}
\hline $\begin{array}{c}\text { Pearson } \\
\text { Correlation }\end{array}$ & $\begin{array}{l}\text { Lysholm Score and VAS } \\
\text { Score }\end{array}$ & ROM and Lysholm Score & $\begin{array}{c}\text { No. of Stitches and Tear } \\
\text { Length }\end{array}$ & $\begin{array}{c}\text { No. of Stitches and Increase } \\
\text { in Joint Space }\end{array}$ \\
\hline$R$ value & 0.746 & 0.694 & 0.853 & 0.142 \\
\hline$R^{2}$ value & 0.556 & 0.481 & 0.728 & 0.02 \\
\hline
\end{tabular}

NOTE. A strong correlation was observed between the number of stitches and the tear length.

ROM, range of movement; VAS, visual analog scale.

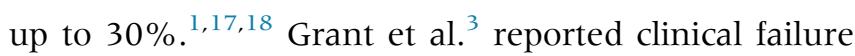
rates of $17 \%$ for inside-out repairs and $19 \%$ for allinside repairs. In studies reporting the mean time to failure, the mean was uniformly greater than 2 years. Our follow-up period was 31.6 months (range, 24-45 months), and we had 1 failure $(2.7 \%)$, in a patient with a high body mass index, which led to partial meniscectomy at the 21 st month. Meniscal repair may give better results and outcomes over meniscal resection when ACLR is performed with a reparable meniscal tear. ${ }^{18,19}$ In our study, no correlation was found between the Lysholm score and patients having undergone ACL repair (or not). In our experience, vertical stitches were more stable than horizontal stitches for meniscal repair, which was also shown in a biomechanical study by Tabrizi and Shariyate, ${ }^{20}$ stating that a vertical loop caused greater stability against tensile force.

In our opinion, pie crusting provided better space to allow the surgeon to work within the operating field and enabled the surgeon to repair the meniscal tear more effectively, with no increased complications. The inside-out technique may have a longer learning curve, but it is more versatile and could be more stable.

\section{Limitations}

This was a retrospective study of a single surgeon's practice without a control group to compare iatrogenic cartilage damage without pie crusting. Only l outcome score (Lysholm score) was obtained at 6 months postoperatively, and not enough postoperative radiologic imaging data were obtained to analyze for more meaningful conclusions. Reporting JSW measurements using a probe might be subject to reporting bias. Finally, the follow-up duration was relatively short to draw conclusions on failure rate.

\section{Conclusions}

In this study, we obtained good outcomes using arthroscopic inside-out medial meniscal repair combined with pie crusting for the release of the MCL, with or without ACLR.

\section{References}

1. Nepple JJ, Dunn WR, Wright RW. Meniscal repair outcomes at greater than five years: A systematic literature review and meta-analysis. J Bone Joint Surg Am 2012;94: 2222-2227.

2. Pujol N, Seil R. Meniscus repair: Updated techniques (open and arthroscopic). In: Hulet C, Pereira H, Peretti G, Denti M, eds. Surgery of the meniscus. Berlin 211-223: Springer Verlag, 2016.

3. Grant JA, Wilde J, Miller BS, Bedi A. Comparison of inside-out and all-inside techniques for the repair of isolated meniscal tears: A systematic review. Am J Sports Med 2012;40:459-468.

4. Jeon SW, Jung M, Chun YM, et al. The percutaneous piecrusting medial release during arthroscopic procedures of the medial meniscus does neither affect valgus laxity nor clinical outcome. Knee Surg Sports Traumatol Arthrosc 2018;26:2912-2919.

5. He X, Cai H, Zhang K. Pie-crusting technique is effective and safe to release superficial medial collateral ligament for total knee arthroplasty. J Orthop Trans 2018;13:33-40.

6. Bert JM. First, do no harm: Protect the articular cartilage when performing arthroscopic knee surgery! Arthroscopy 2016;32:2169-2174.

7. Oakley SP, Portek I, Szomor Z, et al. Arthroscopic estimation of the extent of chondropathy. Osteoarthritis Cartilage 2007; 15:506-515.

8. Flanigan DC, Carey JL, Brophy RH, et al. Interrater and intrarater reliability of arthroscopic measurements of articular cartilage defects in the knee. J Bone Joint Surg Am 2017;99:979-988.

9. Robinson JR, Jermin PJ. Meniscal root tears: Current concepts. Asian J Arthrosc 2016;1:35-46.

10. Beattie KA, Duryea J, Pui M, et al. Minimum joint space width and tibial cartilage morphology in the knees of healthy individuals: A cross-sectional study. BMC Musculoskelet Disord 2008;9:119.

11. Ha CW, Park YB, Lee CH, Awe SI, Park YG. Selective medial release technique using the pie-crusting method for medial tightness during primary total knee arthroplasty. J Arthroplasty 2016;31:1005-1010.

12. Nelson CG, Bonner KF. Inside-out meniscus repair. Arthrosc Tech 2013;2:e453-e460.

13. Chahla J, Cruz RS, Cram TR, Dean CS, LaPrade RF. Inside-out meniscal repair: Medial and lateral approach. Arthrosc Tech 2016;5:el63-el68.

14. Maffulli N, Longo UG, Campi S, Denaro V. Meniscal tears. Advantages of this technique include the ability to achieve consistent perpendicular suture placement through the meniscal tear in the posterior horn. Open Access J Sports Med 2010;1:45-54.

15. Espejo-Reina A, Serrano-Fernández JM, MartínCastilla B, Estades-Rubio FJ, Briggs KK, Espejo- 
Baena A. Outcomes after repair of chronic buckethandle tears of medial meniscus. Arthroscopy 2014;30: 492-496.

16. Fillingham YA, Riboh JC, Erickson BJ, Bach BR, Yanke AB. Inside-out versus all-inside repair of isolated meniscal tears: An updated systematic review. Am J Sports Med 2016:45:234-242.

17. Fuchs A, Kloos F, Bode G, et al. Isolated revision meniscal repair-Failure rates, clinical outcome, and patient satisfaction. BMC Musculoskelet Disord 2018;19: 446.
18. Majeed H, Karuppiah S, Sigamoney KV, et al. All-inside meniscal repair surgery: Factors affecting the outcome. J Orthop Traumatol 2015;16:245-249.

19. Phillips M, Rönnblad E, Lopez-Rengstig L, et al. Meniscus repair with simultaneous ACL reconstruction demonstrated similar clinical outcomes as isolated ACL repair: A result not seen with meniscus resection. Knee Surg Sports Traumatol Arthrosc 2018;26:2270-2277.

20. Tabrizi A, Shariyate MJ. Biomechanical study of meniscal repair using horizontal sutures and vertical loop techniques. Adv Biomed Res 2018;7:144. 